\title{
Agronegocios
}

\section{Definición del enfoque organizacional para una oficina de proyectos agropecuarios en un centro de investigación académica}

Definition of the functional approach for an Agricultural Projects Office in an academic research center

\author{
David Mora Valverde 1 \\ Alejandro Chacón Villalobos²
}

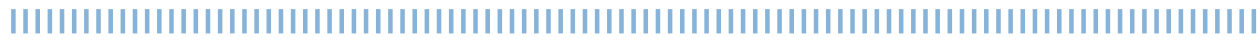

Fecha de recepción: 08 de febrero, 2019

Fecha de aprobación: 20 de junio, 2019

Vol.5 No2 Julio- diciembre 2019

Mora Valverde, D. y Chacón Villalobos, A. (2019). Definición del enfoque organizacional para una oficina de proyectos agropecuarios

en un centro de investigación académica en Costa Rica Revista

E-Agronegocios, 5(2) https://doi.org/10.18845/rea.v5i2.4453.

DOI: https://doi.org/10.18845/rea.v5i2.4453

Ingeniero Agrónomo Zootecnista Universidad de Costa Rica, Costa Rica. Maestría Académica en Ingeniería Industrial Universidad de Costa Rica. Investigador docente de Universidad de Costa Rica. Profesor e investigador en la Estación 


\section{Resumen}

Se efectuó una caracterización de la estructura organizacional y de los principales procesos de un centro de investigaciones público en producción animal dedicado a desarrollar proyectos de investigación agropecuaria, con el objetivo de establecer un marco de referencia que facilite la definición y eventual implementación de una oficina de proyectos adaptable a su quehacer académico.

Palabras clave: Gestión del proyecto, Investigación Operativa.

\section{Abstract}

A characterization of the organizational structure and the main processes of a center dedicated to developing research projects in the agricultural area was carried out, with the objective of establishing a reference framework that facilitates the definition and eventual implementation of a project office adaptable to the center's academic work.

Key words: Project Management, Operations Research. 


\section{Introducción}

Con miras al mejoramiento continuo de los procesos efectuados en el sector académico universitario dedicado a la investigación, se proyecta como necesario el estudio en las diversas técnicas operativo-administrativas que faciliten la consecución adecuada de los proyectos en las instituciones.

Diversidad de centros de investigación, al dedicarse meramente a su objeto de estudio, no necesariamente se acoplan a sistemas administrativos de control de procesos debido a que no son de carácter obligatorio o bien no forman parte de ningún mecanismo de rendición de cuentas. Por otra parte, las limitaciones presupuestarias de estos centros hacen que muchas veces, aunque exista la voluntad, no se orienten recursos hacia el estudio de los procesos, sino al desarrollo de los proyectos de investigación.

Esto permite que se cumpla con la estrategia a la cual se enfocan, pero no se acostumbra justificar, a nivel técnico, la dotación de mecanismos facilitadores no obligatorios para la mejora operativa en la gestión de los proyectos en sí. Es por ello que los centros de experimentación que basan su quehacer en el desarrollo y mantenimiento de proyectos de investigación representan recintos con un potencial de importancia para que la práctica y la teoría de la administración de proyectos juegue un rol determinante.

La complejidad estructural de las organizaciones dedicadas a la investigación y desarrollo hace que se destine naturalmente más recursos sobre los esfuerzos específicos en la resolución de problemas de investigación sin destinar recursos sobre potenciales dinámicas estructuradas propias de la administración de un proyecto de investigación. Lo anterior podría explicarse básicamente en que la formación de los líderes de estos centros se concentra sobre las especialidades técnicas per se, donde puede ignorarse la posibilidad de administración de proyectos, lo cual conlleva a un empirismo en el ejecútese del día a día.

Lo anterior lo recalca Rubio (2009), quien afirma que hacen falta modelos explícitos de estructura organizacional que estén fundamentados con criterios para mejorar los procesos de investigación entre técnicos con el fin de mejorar la productividad de estos centros y de los propios proyectos de investigación con miras a potenciar el impacto de estos sobre la sociedad.

Al ser los proyectos de investigación el principal medio de gestión de resultados, y los productos académicos de éstos el principal medio de proyección de los mismos (artículos científicos, información técnica, asesoría a productores, producción audiovisual, capacitaciones, etc.), la aplicación de las teorías de gestión de proyectos se ajusta adecuadamente en sus diferentes abordajes. Por tanto, se abre la inquietud de que exista en su justa escencia una oficina de proyectos (PMO, Project Management office, por sus siglas en inglés), asi como límites y obligaciones variables según la dinámica del grupo profesional dedicado, para asi otorgar un seguimiento más atento y profesional desde el punto de vista administrativo. 
Las PMO son actualmente una característica prominente de la gestión de proyectos en las organizaciones y la lógica subyacente que conduce a su aplicación o renovación todavía no se contextualiza (Aubry, Hobbs, Müller\& Blomquist, 2010) al menos en un nivel metodológico científico. De igual manera, Hobbs, Aubry\& Thuillier (2008), mencionan que la prevalencia de una $\mathrm{PMO}$ es un fenómeno importante y relativamente reciente. La gran variedad de tipos de PMO y la falta de un patrón de definición claro puede generar confusión a la hora de establecer su arquitectura. Sin ser lo anterior una limitante, cuando una organización identifica las áreas de competencia que le son importantes, puede evitar el desperdicio de recursos en zonas de poca relevancia para lograr la madurez en las zonas que son de alta relevancia, lo cual puede ser aplicable en escenarios dedicados a la producción académica.

Las organizaciones no deberían implementar una PMO bajo el simple supuesto de la relación calidad-dinero o porque las oficinas de proyectos son actualmente populares. Según Pellegrinelli \& Garagna (2009), las PMO deben ser diversas, justificar su valor añadido; se formen, se disuelvan y sean potencialmente reformadas y se espera que funcionen a través del cambio. Estas tienen que demostrar, sobre una base continua, que están haciendo una contribución sustancial al desempeño de la organización a un costo razonable.

Asimismo, muchos líderes están dando el mandato de implementar una PMO sin una imagen clara de lo que esto podría implicar. La dificultad radica en la gran variedad de PMO en la actualidad en el lugar y la falta de consenso en cuanto a su valor (Hobbs et al, 2008).

Tomando en cuenta lo anteriormente expuesto, el presente documento describe la aplicación de un ejercicio de diagnóstico organizacional para un centro académico de investigación agropecuaria como mecanismo para la definición y adecuación de un modelo de PMO en conjunto con la investigación en el estado del conocimiento de las PMO's.

\section{Metodología}

El desarrollo del presente trabajo aconteció en un centro de investigación agropecuario público ubicado en Costa Rica y especializado en el área pecuaria. El estudio tuvo dos fases, la primera correspondió al desarrollo del Modelo Descriptivo Organizacional (MDO), como contexto para la PMO, y la segunda etapa se enfocó en el estudio del componente PMO a partir de la información generada en la primera fase.

El desarrollo del MDO se efectuó a través de entrevistas al personal a cargo de proyectos de investigación y posteriormente a través de un proceso de reuniones semanales de 3 horas durante 3 meses, las cuales permitieron completar el levantamiento de generalidades de la estructura organizacional actual (Jones, 2008), la definición de factores de potencialidad y rezago, el análisis FODA del sector académico y el desarrollo de matrices EFI y EFE para evaluación de factores externos e internos (Ponce, 2007). Para las reuniones semanales se levantaron minutas de registro. Igualmente se desarrolló una prueba ácida de vulnerabilidad del proceso principal con la cual se analizaron cada uno de los pasos de este proceso. 
El Centro de Investigaciones del presente estudio desarrolla una amplia diversidad de proyectos de investigación en producción primaria y secundaria pecuaria enfocándose a un grupo amplio de especies, así como de técnicas agrícolas y de procesamiento. Los temas se desarrollan con un enfoque académico científico, así como de aplicabilidad en campo. De igual forma, entre sus proyectos y desde una perspectiva de enseñanza, la unidad capacita profesionales y estudiantes universitarios con tecnología aplicable a los distintos campos de la producción animal. Para el desarrollo de todas las actividades anteriormente mencionadas, el centro experimental mantiene vigentes y de manera simultánea entre 20 y 25 proyectos, dirigidos por un capital humano variable de entre 7 y 8 investigadores, con un total de 35 colaboradores para totas las actividades de la institución.

El desarrollo del MDO se efectuó a través de entrevistas al personal a cargo de proyectos de investigación y posteriormente a través de un proceso de reuniones semanales de 3 horas durante 3 meses las cuales permitieron completar el levantamiento de generalidades y estructura organizacional actual (Jones, 2008), la definición de factores de potencialidad y rezago, el análisis FODA del sector académico y el desarrollo de matrices EFI y EFE para evaluación de factores externos e internos. Para las reuniones semanales se levantaron minutas de registro. Igualmente se desarrolló una prueba ácida de vulnerabilidad del proceso principal con la cual se analizaron cada uno de los pasos de este proceso.

\section{Resultados y discusión}

Habiendo efectuado el levantamiento descriptivo organizacional con miras a generar recomendaciones para una PMO se obtuvieron los siguientes resultados:

\section{Caracterización organizacional del centro de investigación evaluado:}

Cultura Organizacional: en cuando a este concepto, no se siguen enunciados formales y específicos que sean de conocimiento por parte de los integrantes.

Estructura Organizacional: Según Brito (2016), las estructuras mecánicas son muy centralizadas y formales. Requieren de un alto grado de especialización por parte de los diferentes miembros y se debe mantener una cadena de mando rígida. Por otra parte, las estructuras orgánicas son mucho más descentralizadas y menos formales y especializadas. Esto permite que exista mayor colaboración y participación entre los miembros de la organización. Adicionalmente las estructuras orgánicas son más flexibles y se pueden adaptar a diferentes necesidades que puedan surgir. Para el caso del Centro de investigaciones estudiado presenta una estructura que puede describirse como funcional, con componentes mecanizados y otros orgánicos en los procesos de soporte administrativo y de orgánico, para el proceso de investigación, el cual es el principal.

Para el caso de los componentes mecanizados, esta es una estructura que se diseña para persuadir a las personas a comportarse de manera predecible y responsable (Fred, 2003). Como ejemplos, en el centro de investigación que fue objeto de estudio, se encuentran la 
especialización individual reflejada en el personal de soporte a la investigación (secciones agrícolas/pecuarias y administración), donde los colaboradores trabajan por separado y se especializan en tareas definidas claramente, asimismo posee mecanismos simples de integración donde la jerarquía de autoridad está claramente definida y es el principal mecanismo de integración (como puede observarse en la estructura organizacional actual, la cual es una jerarquía de tres niveles, ver Figura 1). En el rango administrativo y de soporte a la investigación, se identificó la centralización en la autoridad, donde el control para una gran diversidad de tareas externas al proceso central (investigación) se mantiene en el nivel más alto de la organización. La mayor parte de la comunicación es vertical.

Tal y como funciona en este centro de estudio, en muchas organizaciones la selección y prioridad de los proyectos se determina por una Junta de Revisión de Proyectos (Consejo Científico, para este caso). Cada coordinador encargado de un proyecto proporciona información acerca de los recursos a requerir, por lo que el director del centro de investigación determina la viabilidad de realizar cada nuevo proyecto y asignar los recursos a los proyectos y actividades con la más alta prioridad. Lo anterior no necesariamente se efectúa bajo criterios cuantificables de demanda.

Dentro de los principales beneficios de esta figura se encuentra para el caso del mando principal en la institución en estudio, que el compromiso permanente para con la institución y la estructura de mando vertical genera fluidez en el avance principalmente desde el punto de vista de crecimiento institucional. Dentro de los aspectos menos favorables de esta estructura, Stanford (2015), lo caracteriza como una estructura como molinos de viento y lo describe de la siguiente manera, "...divisiones o departamentos que trabajan en forma independiente (...), haciendo menos fluida (...) la cooperación y colaboración con otras funciones de la organización. Existe falta de información compartida entre ellas que puede conducir a la duplicidad, superposición y a una mayor lentitud para conseguir que el trabajo se realice. Asimismo, puede conducir a cometer errores y falta de acción cuando la ocasión lo requiere...".

\section{Caracterización del proceso principal (investigación por proyectos).}

El proceso específico de investigación dentro del centro experimental inicia con la generación de una idea, la cual puede proceder desde un asunto coyuntural o una nueva necesidad encontrada que sea de beneficio del sector agropecuario. A cada una de estas ideas le prosigue una serie de actividades de revisión y ejecución de metas que se detallan en la Figura 1. 


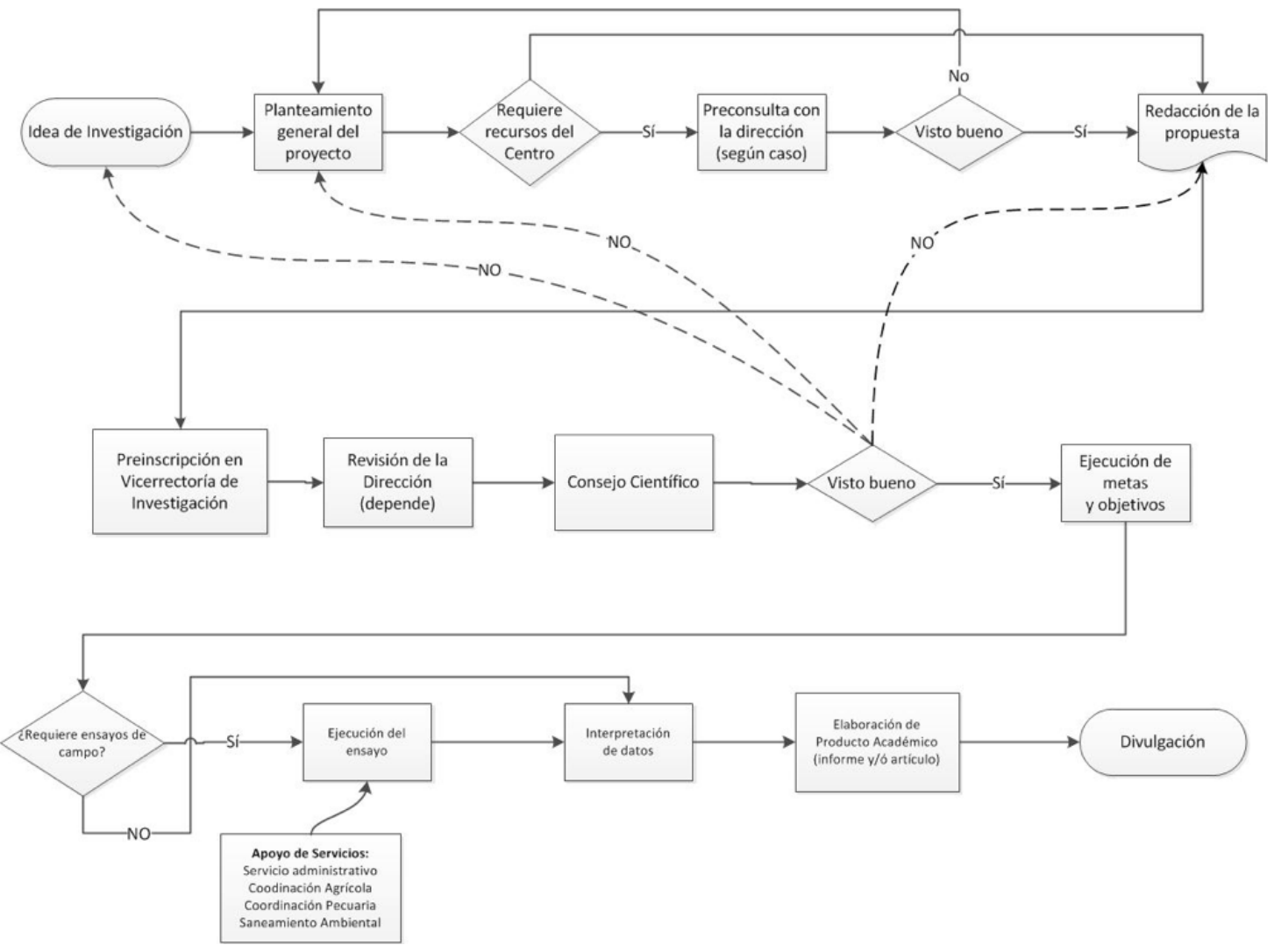

Figura 1. Detalle del proceso de investigación dentro del centro académico evaluado.

Fuente: Elaboración propia.

Dentro de las limitaciones del sistema de organización actual se encuentra que los proyectos están expuestos a barreras existentes entre las funciones jerárquicas de los diferentes grupos de trabajo que la conforman, además de su falta de formalidad organizacional, de tal manera que no necesariamente se tiene consistencia a lo largo del tiempo, ni la flexibilidad que su naturaleza requiere, limitando así el potencial inherente a la capacidad de los investigadores que participan en la realización de tales proyectos (Rubio, 2009).

Otra limitación radica en que los avances de los proyectos de investigación son evaluados de manera anual, lo cual expone las dificultades o retrasos hasta el momento de la evaluación por parte de pares revisores. Lo anterior hace que no se visibilicen las dificultades en tiempo real y no se presione asertivamente sobre el cumplimiento de plazos estipulados, lo que puede provocar un menor aprovechamiento de recursos y tiempo.

Para este sector de la organización, el fenómeno de formación de molinos de viento puede describirse de la siguiente manera "... una percepción estanca, con frecuencia incentivada por la estructura jerárquica y reforzada por los sistemas de incentivos y reconocimiento. Personas con una mentalidad de molino son reticentes a compartir información, a establecer conexio- 
nes entre su trabajo y el trabajo de otros compañeros o departamentos, o a visualizar dónde la colaboración podría implicar un beneficio mutuo..." (Rubio, 2009). Lo anterior se ve reflejado en que existe muy poco intercambio entre investigadores de proyectos diferentes, lo cual puede reducir la potencialización de mejoras en la investigación.

Como resultado del levantamiento de factores de potencialidad y rezago dentro del proceso de proyectos académicos se recabó la siguiente información en términos de factores de potencialidad y factores de rezago, según se resume en el Cuadro 1.

Cuadro 1. Factores de potencialidad y de rezago en el centro de investigación evaluado

\begin{tabular}{|c|c|}
\hline Factores de potencialidad & Factores de rezago \\
\hline $\begin{array}{c}\text { Buenas relaciones entre el sector de } \\
\text { investigadores y el sector administrativo, el cual } \\
\text { gestiona y materializa la consecución de labores } \\
\text { de los proyectos. }\end{array}$ & $\begin{array}{c}\text { Poca planificación integrada } \\
\text { administrativo/académica, lo que afecta el } \\
\text { potencial de impacto y la coordinación. }\end{array}$ \\
\hline $\begin{array}{l}\text { Personal académico capacitado en los diversos } \\
\text { campos del área de impacto. }\end{array}$ & $\begin{array}{c}\text { Instalaciones y/o equipo de investigación } \\
\text { subutilizado que tienen un potencial de generar } \\
\text { mayores resultados académicos }\end{array}$ \\
\hline $\begin{array}{l}\text { Anuencia de los académicos a la } \\
\text { reestructuración organizacional formal y su } \\
\text { correcta aplicación. }\end{array}$ & $\begin{array}{l}\text { Poca coordinación académica inter-proyectos y } \\
\text { escasa retroalimentación entre pares. }\end{array}$ \\
\hline $\begin{array}{l}\text { La unidad académica capaz de potencializar su } \\
\text { imagen como marco de referencia en el sector. }\end{array}$ & $\begin{array}{c}\text { Carencia de una política de planificación } \\
\text { cronológica ni de evaluación formal y propositiva } \\
\text { de resultados. }\end{array}$ \\
\hline $\begin{array}{l}\text { Potencial no aprovechado de alianza con el } \\
\text { sector agroindustrial. }\end{array}$ & $\begin{array}{l}\text { Poca divulgación formal de resultados hacia los } \\
\text { sectores no académicos. Inexistencia de una } \\
\text { instancia a cargo de la divulgación y/o } \\
\text { relaciones públicas (mercadeo y/o ventas). }\end{array}$ \\
\hline
\end{tabular}

Fuente: Elaboración propia.

El desarrollo del ejercicio FODA permitió el desarrollo de las matrices de factores internos y externos (Matriz EFI y EFE), las cuales a través de su ponderación cuantitativa siguiendo la metodología sugerida por Vásquez (J. Vázquez, comunicación personal, 11 de diciembre de 2011) permitió la generación de una matriz de posición estratégica recomendada la cual se detalla a continuación en la Figura 2. La matriz de evaluación de factores internos Matriz EFI es un instrumento para formular estrategias resume y evalúa las fuerzas y debilidades más importantes dentro de las áreas funcionales de un negocio y además ofrece una base para identificar y evaluar las relaciones entre dichas áreas (Fred, 2013). 


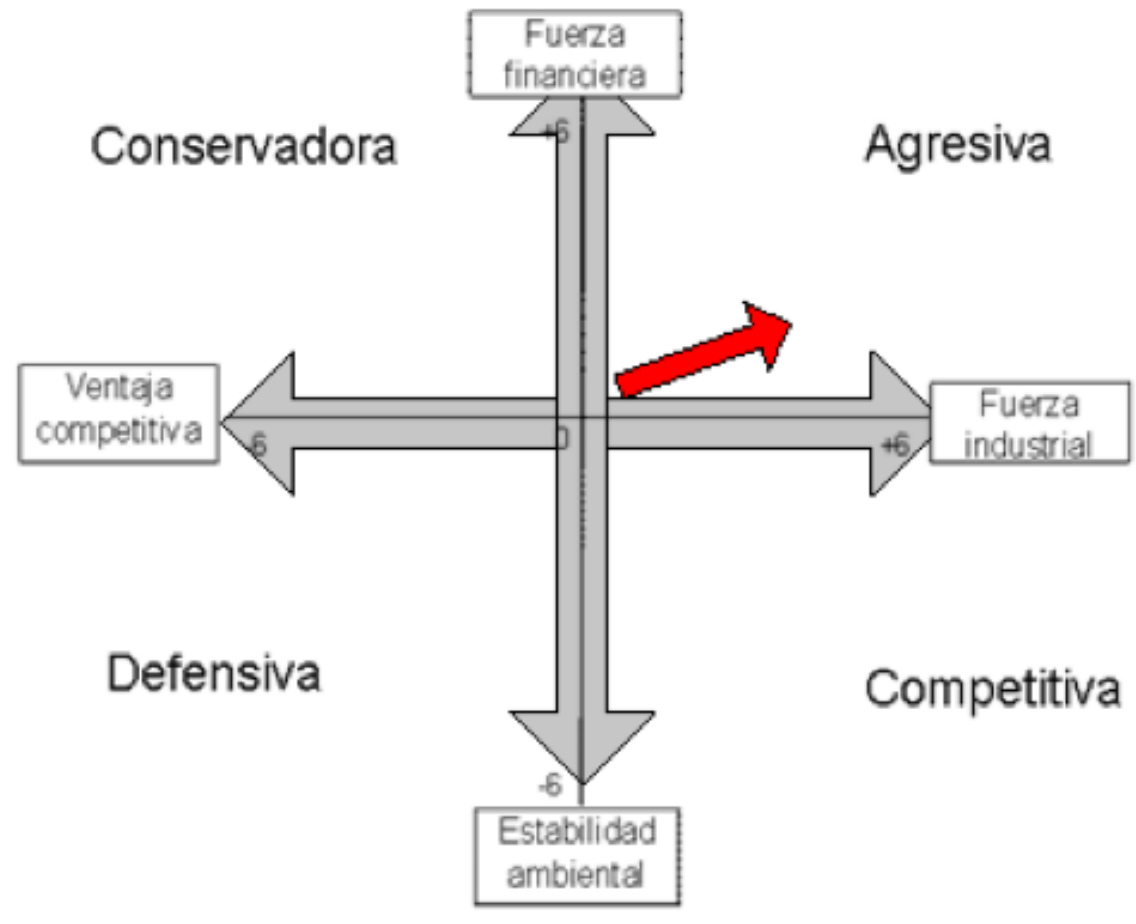

Figura 2. Matriz EFI y EFE para el proceso de investigación dentro del centro académico evaluado.

Fuente: Elaboración propia.

Como resultado de la aplicación de la prueba ácida del proceso, en la cual se califica de manera cuantitativa cada parte del proceso según su importancia mediante valores ponderados, se muestra cómo la dirección del centro de investigación se constituye en un facilitador vital ya que participa en muchas actividades de planificación y guía de este proceso. Su contribución es sumamente importante en la unidad de investigación. De ahí que pueda ser necesario cierta descentralización de la autoridad en la parte administrativa para que se puedan concentrar esfuerzos en la coordinación e implementación académica (Cuadro 2).

Para el caso de la centralización en los procesos, Pellegrinelli \& Garagna (2009), registran que en muchos casos el problema de la centralización es que luego se debe hacer práctica habitual de utilizar en los proyectos y es por eso por lo que muchos descartan la PMO, porque se desea al músculo de la empresa dentro del negocio operativo.

Para el análisis efectuado prácticamente todos los procesos resultaron vitales a excepción de los cuales no aplica la gestión de intermediación de la dirección, pero lo anterior no representa volatilidad para todos los proyectos que requieran de la utilización de la fuerza de soporte del centro de investigación. 
Cuadro 2. Resultado de prueba ácida efectuada al proceso de investigación del centro experimental.

\begin{tabular}{|c|c|c|c|c|c|c|c|c|c|c|c|}
\hline Actividades & $\mathrm{P} 1$ & $\mathrm{P} 2$ & P3 & $\mathrm{P}_{4}$ & P5 & Valor & $T$ & C & $\mathrm{V} / \mathrm{T}$ & $\mathrm{V} / \mathrm{C}$ & Clasificaciór \\
\hline Creación de idea & 1,25 & 1,25 & 1,25 & 1,25 & & 5 & 1 & 1 & 5 & 5 & Vital \\
\hline $\begin{array}{l}\text { Planteamiento de } \\
\text { Proyecto }\end{array}$ & 1,25 & 1,25 & 1,25 & 1,25 & & 5 & 2 & 1 & 2,5 & 5 & Vital \\
\hline Preconsulta al director & 1,25 & 1,25 & 0 & 0 & & 2.5 & 1 & 1 & 2.5 & 2.5 & Vulnerable \\
\hline Redactar el Proyecto & 1,25 & 1,25 & 1,25 & 1,25 & & 5 & 2 & 1 & 2,5 & 5 & Vital \\
\hline $\begin{array}{l}\text { Preinscripción } \\
\text { Vicerrectoría }\end{array}$ & 1,25 & 1,25 & 1,25 & 1,25 & & 5 & 1 & 1 & 5 & 5 & Vital \\
\hline $\begin{array}{l}\text { Revisión de la } \\
\text { Dirección }\end{array}$ & 1 & 1 & 0 & 0 & 1 & 3 & 1 & 1 & 3 & 3 & Vulnerable \\
\hline $\begin{array}{l}\text { Discusión y } \\
\text { Preaprobación }\end{array}$ & 1 & 1 & 0 & 0 & 1 & 3 & 1 & 1 & 3 & 3 & Vulnerable \\
\hline $\begin{array}{l}\text { Consejo científico } \\
\text { aprueba o desaprueba }\end{array}$ & 1 & 1 & 1 & 1 & 1 & 5 & 1 & 1 & 5 & 5 & Vital \\
\hline $\begin{array}{l}\text { Ejecución de Metas y } \\
\text { Objetivos }\end{array}$ & 1,25 & 1,25 & 1,25 & 1,25 & & 5 & 5 & 5 & 1 & 1 & Vital \\
\hline Apoyo y servicios & 1,25 & 1,25 & 1,25 & 1,25 & & 5 & 5 & 5 & 1 & 1 & Vital \\
\hline Recolección de datos & 1,25 & 1,25 & 1,25 & 1,25 & & 5 & 5 & 1 & 1 & 5 & Vital \\
\hline $\begin{array}{l}\text { Interpretación de los } \\
\text { datos }\end{array}$ & 1 & 1 & 1 & 1 & 1 & 5 & 2 & 3 & 2,5 & 1,67 & Vital \\
\hline $\begin{array}{l}\text { Elaboración de } \\
\text { producto académico }\end{array}$ & 1,25 & 1,25 & 1,25 & 1,25 & & 5 & 4 & 5 & 1,25 & 1 & Vital \\
\hline $\begin{array}{l}\text { Generación de nueva } \\
\text { idea }\end{array}$ & 0 & 0 & & 1,67 & & 1,67 & 1 & 1 & 1,67 & 1,67 & Vulnerable \\
\hline
\end{tabular}

Fuente: Elaboración propia.

\section{Sobre adaptar una PMO}

Existen dos estructuras de PMO, la centralizada y la descentralizada. La estructura centralizada, el director de la PMO es quién toma las decisiones de los proyectos y todos los directores de proyectos (investigadores) reportan al director de la PMO. Este tipo de estructura tiene la ventaja de que las instrucciones son aplicadas en una forma más rápida. Pero tiene la desventaja de que el PMO va a ser caracterizada como una "policía de proyectos" provocando que muchos líderes del área funcional dejen de colaborar activamente en proyectos claves (Hauck, 2007).

De manera análoga al sistema centralizado, Lev-Shamur (2009) lo clasifica como un modelo fuerte, donde la PMO se encarga de todos los aspectos del ciclo de vida de un proyecto. El director de la PMO probablemente se encargará de todos los proyectos. Igualmente, resume entre sus ventajas que este modelo tiene mucha influencia en la organización lo cual facilita en la búsqueda de fondos y recursos, puede establecer estándares más altos porque controla directamente todos los proyectos y puede lograr mayor nivel de profesionalismo, ya que todos los miembros son practicantes de proyectos a tiempo completo. Y dentro de las desventajas, se enumera que se requiere un cambio de cultura, altos costos y tiempo para establecer los requerimientos estándares, herramientas y miembros, así como la resistencia al cambio y un aumento en el nivel de burocracia. 
En una estructura descentralizada Hauck (2007), indica que el PMO tiene la función de ser fuente de consulta para estándares en metodología de gerencia de proyectos y para resolver problemas de proyectos. La anterior perspectiva es análoga con de Lev-Shamur (2009), quien considera de manera similar a la estructura descentralizada, pero la califica como un "modelo consultor", el cual se inclina más sobre educar los gerentes de proyectos y su equipo y dar soporte cuando sea necesario. Puede involucrarse en el establecimiento de estándares, pero desde el punto de vista de recomendación. Entre sus ventajas, afirma que no requiere de extensa reorganización, genera una menor resistencia y puede proveer reportes independientes para la gerencia. Entre las desventajas enumera que este departamento difícilmente tenga acceso a recursos suficientes y pueda dar la impresión de sobrecargarse de trabajo.

Otro modelo reportado también por Lev-Shamur (2009) es el modelo mixto, el cual posee parte de los dos modelos anteriormente mencionados. Este modelo puede aplicar el control de un modelo fuerte, pero con una pequeña cantidad de personas a cargo, normalmente un solo director de PMO. Esta persona sería quién se encarga de establecer los estándares, aprobar y dar reportes.

Una PMO puede ser fácilmente desacreditada cuando se tiene la percepción equivocada de los proyectos. En un caso reportado por Hobbs et al. (2008) una PMO fue desmantelada debido a su incapacidad para ayudar a los gerentes de proyectos, debido a una mala interpretación de las necesidades de los proyectos. Una matriz de factores externos e internos permite cuantificar aspectos relevantes como guía para una dirección de proyectos basándose en el ambiente generalizado de un grupo de trabajo, lo cual permite dejar de lado el uso del empirismo para decisiones de este tipo. Por ejemplo, el factor encontrado "no existe en el departamento una política de planificación cronológica ni de evaluación formal y propositiva de resultados", permite guiar ya sea a un gerente de proyectos a un grupo colegiado a ejercer políticas o estrategias que persigan el mejorar este aspecto. Básicamente lo que permite es conocer las necesidades de los diferentes grupos y subgrupos de producción académica de una manera adecuada.

En algunos casos, la creación de una PMO podría quitar algún grado de autonomía a los encargados de proyectos y un poder de los patrocinadores y gerentes de línea, llevando algunas de las decisiones a un centro director. Lo anterior suscita debates políticos. La experiencia indica que algunos encargados de proyectos son personas que normalmente valoran la autonomía y perciben la normalización de manera desagradable y se podría esperar una resistencia a la disciplina y la "interferencia" impuesta por una PMO Pellegrinelli \& Garagna (2009). Para el caso del centro de investigación, entre las entrevistas no existió una reticencia a que existan mecanismos estandarizados para darle mayor seguimiento a los proyectos, debido a que lo anterior puede generar cierta colaboración cuando existan complicaciones u orientación adecuada. Asimismo, existe disposición a convivir con lo anterior siempre y cuando se respete el principio de creatividad y libertad de cátedra del investigador que para este caso todos son también docentes universitarios. Por lo tanto, sin una adecuada utilización de herramientas de levantamiento descriptivo organizacional difícilmente se hubiese hecho evidente una nece- 
sidad como la mencionada.

Para implementar o bien modificar una PMO, esto debe coincidir con cambios estratégicos provenientes desde la alta dirección. En las experiencias que abordan transformaciones de $\mathrm{PMO}$, los cambios en la estructura coinciden con cambios en la filosofía de la gestión. La filosofía de gestión puede ser relativa de un individuo, del equipo de alta dirección o de toda la organización. Una nueva filosofía de gestión por lo general inicia un proceso de reestructuración, cuando la estructura actual no es coherente con la nueva filosofía de gestión (Hobbs et al, 2008).

Para el rol de gestión de recursos, Pellegrinelli \& Garagna (2009), reportan que un problema común en el proyecto de las organizaciones de base es que proyectos individuales simplemente no tienen los recursos adecuados. Esto ocurre cuando los proyectos son aprobados e iniciados sin considerar los recursos que necesitan en comparación con los recursos disponibles. Como resultado, los recursos se desplazan de un proyecto a otro, y algunos de éstos se retrasan o se aplazan para que otros puedan ser iniciados o terminados a tiempo. Un papel de una $\mathrm{PMO}$ es mantener un registro de los recursos del proyecto, tales como el número de los empleados a tiempo completo en cada puesto de trabajo o categoría de grado de habilidad.

La literatura también considera importante informar la evolución de los proyectos bajo un mecanismo que no intente influenciarlos y donde se evite la confusión causada por diferentes formatos o tipos de informes elaborados por distintos directores de proyectos, con jergas variadas, este tipo de PMO es nombrado por Nicholas \& Steyn (2012) como una "Estación de Control de Proceso Planta Química" y se considera la figura más adecuada para facilitar la evolución de los proyectos.

\section{Conclusiones}

El implementar una oficina de proyectos dentro de este centro de investigación se justifica debido a que el control de los avances de estos proyectos se efectúa en lapsos de tiempo extensos (anualmente). El establecer una oficina de proyectos (PO o PMO) puede ayudar en su gerencia, control, análisis de riesgo, tener las herramientas y técnicas necesarios y los recursos humanos adecuados y atender eventualidades debidas a situaciones no predeterminadas en el ejercicio del planeamiento o ejecución.

Es posible recomendar una PMO también debido a que el ejercicio abierto y continuo de una oficina de proyectos puede aflorar necesidades a todo el grupo de manera constante, permitiendo la inter-colaboración de los proyectos de manera más formal. Dado que un proyecto es verificado prácticamente hasta que completa su ciclo de vida, allí es donde una oficina de proyectos puede ayudar: a supervisar el cumplimiento de este ciclo de vida y de guiar los gerentes de proyectos a cumplir con sus funciones del proyecto. Siempre ofreciendo soporte en la parte de control y mejora continua.

Uno de los modelos más adecuados para aplicar en el centro de investigación es el modelo 
de consultor, ya que el modelo fuerte o mixto, sin ser formalmente establecido como una $\mathrm{PMO}$, se asemeja más a la manera en que actualmente opera el centro, el cual es gestionado por su director. Esto hace que todos los procesos de los proyectos ralenticen su accionar e imposibilita tener una reacción rápida al cambio. Además, para un PMO pequeña, el modelo fuerte requeriría mayores recursos y personal de trabajo, lo cual es algo que las posibilidades del centro no permiten.

\section{Literatura citada}

Hauck, Cynthia. (2007). How to Choose the Right Project Management Office Structure for Your Organization's Culture. Collegiate Project Services. 1-3.

Hobbs, Brian; Aubry, Monique; Thuillier, Denis. (2008). The project management office as an organizational innovation. International Journal of Project Management. 26, 547-555.

Jones, Gareth. (2008). Teoría Organizacional: Diseño y Cambio en las Organizaciones. México: Pearson Hall. Lev-Shamur, Gilad. (2009). PMO series-part 2: Shape your PMO structure. Recuperado el 13 febrero 2018. http://giladlsh.wordpress.com/2009/10/19/pmo-series-part2-shape-your-pmo-structure/.

Nicholas, John; Steyn, Herman. (2012). Project Management for Engineering, Business and Technology. Londres y Nueva York: Routledge.

Rubio, Felipe. (2009). La Estructura Organizacional en Centros de Investigación, Desarrollo e Innovación; una Aproximación a la Experiencia Internacional. Tesis de Doctorado en Administración, Universidad Autónoma de Querétaro, Santiago de Querétaro, México.

Aubry, Monique; Hobbs, Brian; Müller, Ralf; Blomquist, Tomas. (2010). Identifying forces driving PMO changes. Project Management Journal. 41(4), 30-45.

Pellegrinelli, Sergio; Garagna, Luciano. (2009). Towards a conceptualisation of PMOs as agents and subjects of change and rewal. International Journal of Project Management. 27. 649-656. Ponce, Humberto. (2007). La matriz FODA: Alternativa de Diagnóstico y Determinación de Estrategias de Intervención en Diversas Organizaciones. Enseñanza e Investigación en Psicología. 12 (1), 113-130.

Stanford, Naomi. (2015). The Economist Guide to Organisation Design 2nd edition: Creating high-performing and adaptable enterprises. Economist Books 